\title{
INTENSITY-DEPENDENT AUDITORY EVOKED POTENTIAL DEFINES SUBGROUPS OF PATIENTS WITH PTSD: A MulTIMODALITY IMAGING STUDY
}

\author{
Teresa J. Poprawski ${ }^{1,2}$, Kara A. Lonser ${ }^{2}$, John Korpics ${ }^{1,2}$, Jerzy Zadecki ${ }^{2}$, John W. Crayton ${ }^{1,2}$, Angelos \\ Halaris $^{1,2}$, Lukasz M. Konopka ${ }^{1,2,3^{*}}$ \\ ${ }^{I}$ Department of Psychiatry and Behavioral Neuroscience, Loyola University Stritch School of Medicine and Loyola University Medical \\ Center, Maywood, IL USA \\ ${ }^{2}$ Section of Clinical Neuroscience, Edward Hines Jr. VA Hospital, Hines, IL, USA \\ ${ }^{3}$ The Chicago School of Professional Psychology, Chicago Il USA \\ Received March 24, 2009; accepted April 10, 2009
}

\begin{abstract}
Post Traumatic Stress Disorder (PTSD) is a complex and heterogeneous psychiatric disorder. Patients with chronic PTSD present with significant psychiatric co-morbidities. Current treatment guidelines recommend the use of selective serotonin reuptake inhibitors in PTSD patients, but only $50-60 \%$ of these patients respond favorably. To improve the response rate, understanding the underlying pathophysiology is necessary. Serotonergic transmission has been implicated in PTSD and the intensity-dependent auditory evoked potential (IDAEP) is presumed to reflect central serotonergic activity, thereby providing an opportunity to more precisely characterize PTSD patient populations and possibly predict response to treatment. We studied nineteen patients diagnosed with PTSD, matched for age and medications. Based on the stimulus intensity relationship, the patients were divided into two groups: augmenters and reducers. These groups were further evaluated using resting state quantitative EEG and HMPAO-based SPECT brain perfusion acquired simultaneously. The imaging data were correlated with behavioral measures characterizing symptoms of PTSD and measurements of mood state. The augmenters differed from the reducers in the resting state quantitative EEG and SPECT perfusion measures. The differences were primarily localized to the right hemisphere. There were significant differences in measures of PTSD symptoms, but not in overall measures of depression. Item analysis of depression measures showed a significant difference between the augmenters and the reducers in reported sleep difficulties, which correlated with the reported anxiety measures. Objective separation of patients into subgroups based on the IDAEP contributes to the understanding of PTSD biological substrate and can potentially lead to more effective treatment strategies.
\end{abstract}

Key words: EEG; Post-traumatic Stress Disorder; Intensity-dependent Auditory Evoked Potential; SPECT

\section{INTRODUCTION}

Dysregulation of central serotonergic activity has long been implicated in patients with Post-Traumatic Stress Disorder (PTSD) (Arora, Fichtner, O'Connor, \& Crayton, 1993; Harvey, Naciti, Brand, \& Stein, 2004; Southwick et al., 1999). Guidelines of the American Psychiatric Association recommend selective serotonin reuptake inhibitors (SSRIs) as the first-line treatment for patients with PTSD (APA Practice Guidelines, 2004; Foa et al., 1999). However, the response rate is highly variable, suggesting that the PTSD patient population may consist of subgroups with different underlying pathophysiology. Therefore, assessment of serotonergic activity in the central nervous system could

\footnotetext{
* Correspondence to: Lukasz Konopka, Chicago Il USA,

e-mail: lkonopka@thechicagoschool.edu
}

potentially differentiate PTSD patient subgroups. A clinically useful tool reflecting central serotonergic activity is measurement of the intensity-dependent auditory evoked potentials (IDAEP) (Juckel, Hegerl, Molnar, Csepe, \& Karmos, 1999; Juckel, Molnár, Hegerl, Csépe, \& Karmos, 1997). Studies with depressed patients have shown favorable responses to SSRIs in patients showing increased amplitude of the $\mathrm{N} 1 / \mathrm{P} 2$ component of auditory evoked potentials in relation to acoustic stimuli intensity (Hegerl, Gallinat, \& Juckel, 2001). There are only limited data on IDAEP measures in PTSD patients and no studies correlating the clinical response to SSRIs with IDAEP responses in PTSD patients. 
Existing studies have identified two patterns of the N1/P2 response component of auditory evoked potentials to increasing intensity of acoustic stimuli (Lewine, 2002; McPherson, Newton, Ackerman, Oglesby, \& Dykman, 1997; Metzger et al., 2002; Paige, Reid, Allen, \& Newton, 1990). The response patterns have been described as augmentation and reduction (Buchsbaum, 1968). Augmentation is the tendency of the amplitude of the N1/P2 component of auditory evoked potentials to increase as a function of stimulus intensity, while the reverse occurs in reduction. Augmenters and reducers process sensory information differently, as evidenced by the IDAEP. We hypothesized that the difference in sensory processing may reflect differences in resting brain functioning. Four studies have assessed brain function at rest by evaluating regional cerebral blood flow $(\mathrm{rCBF})$ in PTSD (Bonne et al., 2003; Lucey et al., 1997; Mirzaei et al., 2001). No studies have attempted to correlate the different cortical IDAEP responses to baseline brain metabolism. Bonne and colleagues (2003) have compared rCBF between trauma-exposed individuals with and without PTSD. When compared to the nonPTSD group, the PTSD group demonstrated increased $\mathrm{rCBF}$ in the cerebellum, the right precentral superior temporal and the fusiform gyri, and in the left inferior temporal and the supramarginal gyri. In another study, the PTSD group showed a decrease in perfusion ratios in the bilateral frontal cortex and right caudate relative to the healthy control group (Lucey et al., 1997). Mirzaei and associates (2001) examined the rCBF in 8 individuals diagnosed with PTSD and 8 healthy controls and found that the individuals with PTSD had more heterogeneous perfusion in the left hemisphere versus the right hemisphere, when compared to controls. Sachinvala and colleagues (2000) also have reported differences between PTSD patients and controls. The results indicated a significant increase in brain perfusion in subjects diagnosed with PTSD than in the normal controls. The findings were localized to the anterior and posterior cingulate bilaterally, the right temporal and parietal cortex, the right caudate and putamen, and the left orbital and hippocampal regions. In all of these studies, PTSD was a behaviorally diagnosed disorder and subject selection was based on a clinical interview and/or behavioral measures assessing PTSD symptom severity. Discrepancies in the reported findings could be due to the existence of heterogeneous PTSD patient populations. Exclusive use of behavioral measures may lack sufficient sensitivity to detect differences in the pathophysiology of PTSD. In designing this study, we included objective biological measures in an effort to detect the presence of such differences. We divided the PTSD patient population into two biologically distinct subgroups based on the IDAEP patterns: augmenters and reducers. We hypothesized that different processing of the IDAEP reflects differences in the underlying brain abnormalities in PTSD patients. We also hypothesized that neuropathophysiological differences would be reflected in the resting qEEG measures, SPECT brain perfusion, measures of mood, and PTSD symptom intensity.

\section{METHODS \\ Subjects}

This was a naturalistic study. Participants were selected from patients with combat-related PTSD, diagnosed by the DSM-IV criteria. Patients were referred by the treating clinicians for assessment due to an inadequate or lack of response to treatment. The patients were given the Clinician Administered PTSD Scale (CAPS) (Blake et al., 1990) and the Mississippi Scale for Combat Related Post-Traumatic Stress Disorder (M-PTSD) (Keane, 1988). Prior to enrollment, all subjects were informed of the nature and purpose of the study and signed an informed consent form. The Institutional Review Board approved the study.

Forty-three patients diagnosed with PTSD based on positive scores on CAPS, and M-PTSD were enrolled in the study. Of these, twenty-four were excluded from the study (see below). The final group of participants consisted of 19 males ranging in age from 42 to 57 years (mean age of 49.95, $\mathrm{SD}=3.291$ ) and included different ethnic groups: Caucasian $(\mathrm{n}=12)$, African American $(n=5)$ and Latino $(n=2)$. Seventeen subjects were righthanded and two were left-handed. All participants completed functional brain imaging studies including qEEG, IDAEP and SPECT, which were all performed on the same day. In addition, the subjects completed the Beck Depression Inventory (BDI) (Beck, Ward, Mendelson, Mock, \& Erbaugh, 1961) and the State-Trait Anxiety Inventory (STAI) (Spielberger, Gorsuch, \& Lushene, 1970) on the day of the qEEG, IDAEP, and SPECT studies. Since this was a naturalistic study, our population sample closely reflected the type of PTSD patients seen in our clinics. Thus, the presence of medications and co-morbidities was not an exclusion criterion. The study included PTSD subjects with depression and anxiety, as well as patients with a history of alcohol and drug use who did not meet the criteria for dependence at the time of the study. Individuals who tested positive for alcohol or illicit drugs on urine toxicology screening on the test day were excluded from the study. We also excluded patients with an Axis I diagnosis of schizophrenia or any other psychotic disorder. Patients with a history of severe traumatic brain injury causing a structural brain lesion, as confirmed by magnetic resonance imaging (MRI), history of tumor, epilepsy, stroke, or other neurological condition associated with structural brain lesions determined by self-report and/or chart review, were also excluded. Certain coexisting medical conditions were not exclusion criteria. Four subjects had di- 
abetes mellitus; three, hypertension; two, hyperlipidemia; one, arthritis; and one, hepatitis C. All subjects were receiving one or more psychotropic and nonpsychotropic medications at the time of the study. To control for potentially confounding medication effects on the test measures, the final study groups (augmenters and reducers) consisted of patients taking the same class of psychotropic medications.

\section{EEG Data Acquisition}

On the day of testing patients were administered a urine toxicology screen. One subject who tested positive was excluded from the study. The EEG was recorded while the subjects sat with their eyes closed in a reclining chair located in a Faraday Chamber in a specially designated laboratory. Their state of alertness was tracked with EEG. A video camera was used to monitor the subject's behavior. During the recording, the HMPAO ligand (23-25mCi) for brain SPECT imaging was administered via an established IV line outside of the Faraday Chamber. The patients were unaware when the administration of the injection occurred.

The EEG was recorded from 19 cephalic electrodes that were placed according to the International 10/20 System of Electrode Placement. Four non-cephalic electrodes were used to monitor the EKG and eye movements. The electrode impedance was $<5 \mathrm{kOhm}$. The data was acquired with a sampling rate of $500 \mathrm{~Hz}$ with filter settings at $0.1 \mathrm{~Hz}$ and $70 \mathrm{~Hz}$. The earlobe electrodes were used as the active electrode, with a nose electrode as reference. The post-acquisition, linked-ears reference was computed. The awake, eyes closed and eyes open EEG was recorded, and the event-related potentials were recorded at the end of the session.

\section{Auditory Evoked Potentials Acquisition and Processing}

During testing, the subjects were instructed to listen to tones while sitting comfortably with eyes closed. An auditory stimulus of $2000 \mathrm{~Hz}$ was presented binaurally in a pseudo-randomized fashion with an ISI of 1500$2000 \mathrm{~ms}$ through headphones. Each stimulus was of $50 \mathrm{~ms}$ duration with $10 \mathrm{~ms}$ rise and $10 \mathrm{~ms}$ fall time. Stimulus generation and presentation was through the Stim software. A total of 300 tones were administered, 60 at each intensity $(60,70,80,90$, and $100 \mathrm{~dB})$. The auditory evoked response (AER) was extracted from continuous EEG and the files were analyzed in NeuroScan.

Each participant's AER file was re-referenced to linked ears. Epochs of $500 \mathrm{msec}$ duration for each electrode were averaged for each of the five intensities $(60,70$, 80,90 , and $100 \mathrm{~dB}$ ). Visual analysis of the individual epochs resulted in the elimination of data contaminated by artifact and the remaining 35-50 responses were averaged. The averaged amplitude of the N1/P2 compo- nent was measured at $\mathrm{Cz}$. The results were analyzed using SigmaPlot 7.0 (2001). In SigmaPlot, correlation plots (amplitude vs. stimulus intensity) were generated for each participant. The slopes of the correlation defined two groups (Augmenters positive slope; Reducers, negative slope). These data were used to subgroup the patient populations as augmenters and reducers and served as basis for further analysis.

\section{EEG Data Processing}

NeuroGuide 1.8.1 EEG software was used to process EEG data. For the analysis, artifact-free epochs of equal duration between the subjects, containing an eyes closed, relaxed, alert state of EEG record were manually chosen. Split-half reliability tests were conducted on selected segments of EEG and the average electrode split-half reliability was greater than $95 \%$.

NeuroStat, a component of NeuroGuide, was used for the statistical analysis of EEG background activity. The independent t-test was applied to detect statistically significant differences $(\mathrm{p}<0.05)$ in absolute and relative power of the background activity between the two groups: Augmenters and Reducers. $1-\mathrm{Hz}$ frequency bands were computed across all frequency bands (1$30 \mathrm{~Hz})$.

\section{Low-Resolution Electro-Magnetic Tomography (LORETA)}

The NeuroGuide EEG edited files were converted into a format compatible with Key Institute's software, LORETA(Pascual-Marqui, 1994), which is 256 blocks of ASCII data (2 seconds X 128 samples/sec) in which the 19 channels were columns and the 256 time points were rows. LORETA statistic was computed using the Key Institute "FileNames" Maker program to create Augmenters group of 10 subjects and Reducers group of 9 subjects. The non-parametric voxel-wise independent ttest was computed using log-transform and subjectwise normalization. We considered findings as significant at the level of \pm 2.0 standard deviations.

\section{SPECT Imaging Acquisition and Processing}

During the EEG recording a $23-25 \mathrm{mCi}$ of the HMPAO ligand for brain SPECT imaging was administered intravenously. Approximately 90 minutes after the ligand injection, SPECT data acquisition was performed using a rotating gamma camera system equipped with an ultra-high resolution fan beam collimator (Trionix system) for SPECT data acquisition. The images were obtained in a $256 \times 128$ pixel format with an acquisition time of 50 seconds per step. We converted the fan beam projection data to parallel data in a $128 \times 128$ pixel format, and performed the SPECT reconstruction with a Henning filter with a 0.9 -cycles/cm cutoff. Approximately 60 transverse slices were generated. 
A

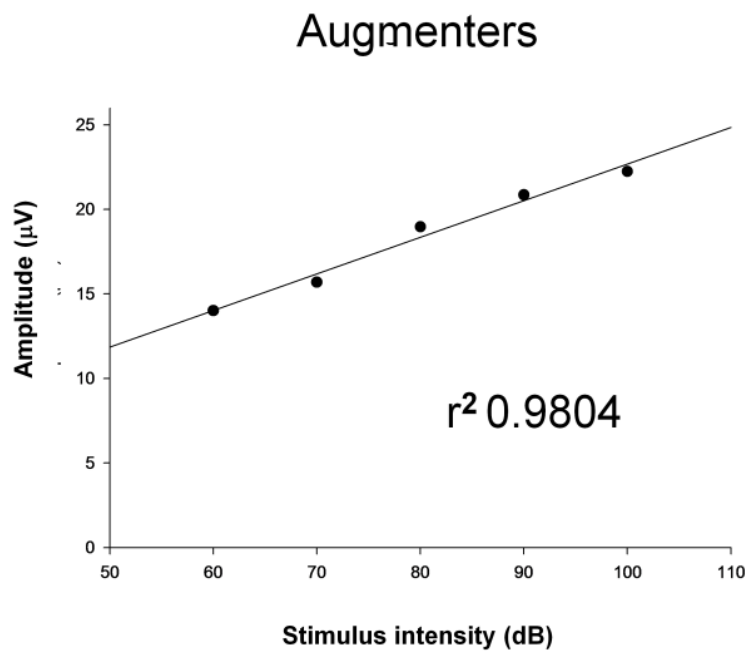

B

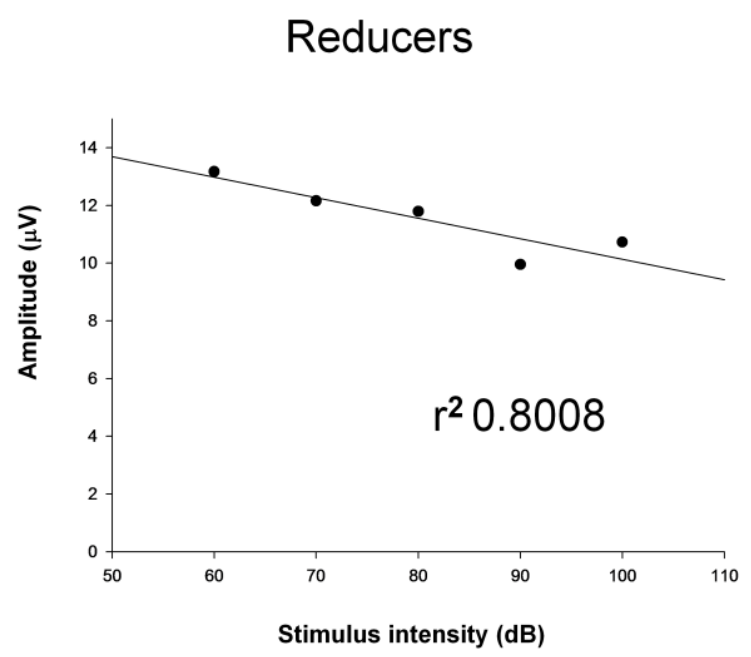

Figure 1. Mean N1/P2 amplitudes $(\mu \mathrm{V})$ plotted as a function of auditory stimulus intensity. A- Augmenters showing increasing response intensity as a function of stimulus intensity. B- Reducers showing decreasing response intensity as a function of stimulus intensity.

Brain SPECT scans were viewed and processed with the MEDx visualization and analysis software program, SPM99 (MEDx 3.4.3, 1999). Each individual's raw brain SPECT image was viewed for any abnormalities (i.e., poor resolution, damage to file, or gross abnormalities) before it was included in the analysis. These images were further analyzed in SPM'99 (Statistical Parametric Mapping), software incorporated into the MEDx program. The images were normalized to a standard space based on the Talairach system (Talaraich \& Tournoux, 1988). Gaussian Smoothing was applied with the filter set at $8 \mathrm{~mm}$ after spatial normalization was completed. The statistical analyses computing voxel by voxel differences between Augmenters and Reducers were processed using an independent t-test.

The statistical analysis included global normalization to adjust for the scan-to-scan differences in global blood flow using proportional scaling. Proportional scaling reduced each voxel value for each scan to the mean. The proportional global mean was set to 50 . This scaled the global blood flow to a physiologically realistic value of $50 \mathrm{ml} / \mathrm{dl} / \mathrm{min}$ (MEDx 3.4.3, 1999). Voxels that failed to reach the specified threshold $(80 \%)$ were excluded to ensure only grey matter voxels were included in the analysis. This process computed the overall mean and then excluded voxels, which did not reach the threshold of the overall mean.

Two contrasts were examined to evaluate increased and decreased rCBF in Augmenters in comparison to Reducers. The height threshold of the SPM program was set at $p=0.02$. The number of voxels to restrict the minimum cluster size was set at 0 , so as to include single voxel clusters. The anatomic location of each significant cluster was determined in the Talairach space by $\mathrm{x}, \mathrm{y}$, and $\mathrm{z}$ coordinates.

\section{RESULTS \\ Auditory Evoked Potentials}

The analysis of the N1/P2 component of the auditory evoked potentials, at each stimulus intensity of the 42 subject records revealed two distinct response patterns. Twenty-nine subjects showed an increase in response intensity as a function of stimulus intensity. We categorized these patients as augmenters. Ten patients showed decreasing responses to increasing stimulus intensity. This group was defined as reducers. The quality of EEG data in three subjects did not allow for the generation of evoked potentials. These patients were not included in the final analysis. To control for medication effects on the IDAEP, the final groups of augmenters and reducers were created after adjusting for this confounding variable. The two groups were matched so subjects in each group were taking the same type of psychotropic medications, which included anxiolytics, antidepressants, and anticonvulsants, and non-psychiatric medications, such as statins, antihypertensive, and antidiabetic medications. The final matched groups consisted of 10 augmenters Augmenters and 9 reducers Reducers. The averaged waveform amplitudes for each tone relative to the tone intensities were plotted for both groups as shown in Figure $1 \mathrm{~A}$ and $\mathrm{B}$. The mean amplitude values at each tone intensity for Augmenters and Reducers are shown in Table 1. 
Table 1. Augmenters and Reducers values of mean N1/P2 amplitude $(\mu \mathrm{V})$ responses at each auditory stimulus intensities. Asterisk $\left(^{*}\right)$ represents measures that reached statistical significance $(\mathrm{P}$ value $<0.01)$.

\begin{tabular}{|l|l|l|l|l|l|}
\hline & $60 \mathrm{~dB}$ & $70 \mathrm{~dB}$ & $80 \mathrm{~dB}$ & $90 \mathrm{~dB}$ & $100 \mathrm{~dB}$ \\
\hline $\begin{array}{l}\text { Augmenters } \\
(\mathrm{n}=10)\end{array}$ & $14.00 \pm 2.36$ & $15.70 \pm 2.69$ & $18.97 \pm 2.87^{*}$ & $20.86 \pm 5.52^{*}$ & $22.24 \pm 6.58^{*}$ \\
\hline Reducers $(\mathrm{n}=9)$ & $13.17 \pm 5.76$ & $12.16 \pm 6.57$ & $11.79 \pm 7.38^{*}$ & $9.95 \pm 5.51^{*}$ & $10.72 \pm 6.21^{*}$ \\
\hline
\end{tabular}

\section{Background EEG}

The quantitative analysis of the background activity demonstrated statistically significant differences in the absolute power between Augmenters and Reducers. These findings were limited to the right hemisphere. Specifically, the Augmenters showed increased theta absolute power in the right temporo-central brain regions: $(\mathrm{T} 4, \mathrm{p}<0.025)(\mathrm{C} 4, \mathrm{p}<0.025)(\mathrm{Cz}, \mathrm{p}<0.035)$.

More precise localization of the increased absolute power of electrical activity in Augmenters relative to Reducers was seen when Low-Resolution ElectroMagnetic Tomography was used. Statistically significant findings were observed in the theta and alpha bands with a peak frequency of $6 \mathrm{~Hz}$ at $\mathrm{z}=3.88$ localized to the postcentral gyrus in the parietal lobe, Broadman's areas: BA 3, BA 1 and BA 2. Increased electrical brain activity was found at $3 \mathrm{~Hz}(\mathrm{z}=2.02)$ and $4 \mathrm{HZ}(\mathrm{z}=2.23)$ localized to the middle temporal gyrus (BA 21, BA 37); at $5 \mathrm{~Hz}(\mathrm{z}=3.15)$ in the insula (BA 13, BA 40) and the superior temporal gyrus (BA 42), at $6 \mathrm{~Hz}(\mathrm{z}=3.88)$ localized to the postcentral gyrus (BA 3, $\mathrm{BA} \mathrm{1,} \mathrm{BA} \mathrm{2);} \mathrm{at} 7 \mathrm{~Hz}(\mathrm{z}=3.82)$ localized to the precental gyrus (BA 4, BA 6) in the frontal lobe, and at $8 \mathrm{~Hz}$ $(\mathrm{z}=2.85)$ and $9 \mathrm{~Hz}(\mathrm{z}=2.33)$ in the middle frontal gyrus (BA 8, BA 9), Figure 2B.

In an attempt to find converging findings with SPECT imaging in the area of increased perfusion seen in Augmenters, we adjusted the level of significance from 3.88 to 3.0. This allowed us to demonstrate increased theta band activity at $6-7 \mathrm{~Hz}$ localized to the bilateral superior frontal (BA 6) and medial frontal gyri (BA 8 and BA 9) as well as to the cingulate (BA 32, BA 24 and BA 33) bilaterally, as shown in Figure 3.

\section{SPECT SPM}

There were statistically significant findings in the $\mathrm{rCBF}$ at the baseline between Augmenters and Reducers (at the threshold $\mathrm{z}>2.8, \mathrm{p}=0.02$ ). The majority of findings were localized to the right hemisphere. The three significant clusters of decreased $\mathrm{rCBF}$ were identified in Augmenters compared to Reducers (Table 2). The clusters were localized to the right inferior, middle, the superior temporal gyri and the insula. These findings colocalized with the qEEG findings (Figure 2A).

Augmenters showed a significantly increased $\mathrm{rCBF}$ in comparison with Reducers (Table 2). The findings in the left hemisphere include two suprathreshold clusters, which were localized in the middle frontal gyrus (BA 6). Augmenters were also characterized by increased activity in the right superior, medial frontal gyri and the cingulate (Figure 3). These rCBF findings co-localized with the qEEG findings of increased theta activity as shown in Figure 3.

\section{Behavioral Measures}

Augmenters group had a greater severity of PTSD symptoms as rated by CAPS and M-PTSD. Augmenters and Reducers were also significantly different with respect to the subscales of CAPS (re-experiencing, CAPS$\mathrm{B}$; avoidance/numbing, CAPS-C; and increased arousal, CAPS-D). Augmenters appear to be more affected across all domains of the PTSD symptom spectrum.Total BDI scores, as a measure of depression, did not yield statistically significant differences between Augmenters and Reducers. Phenomenologically, PTSD shares symptoms with depressive disorder. We postulated that there might be differences in these two populations in the endorsement of somatic symptoms. To address this issue we conducted an item analysis of BDI variables. Reducers reported significantly more sleep difficulties than Augmenters (BDI item 16). We also explored the possibility that the increased sleep difficulties in Reducers could be associated with increased anxiety in this population. STAI-T scores in Reducers were significantly higher than those in Augmenters (Table 3).

\section{DISCUSSION}

The goal of this study was to differentiate PTSD patient populations based on the auditory intensity paradigm. We postulated that the baseline measures acquired at rest (qEEG and SPECT) would support the differences seen in the IDAEP. As hypothesized, this objective approach allowed us to subdivide PTSD subjects into two distinct groups: Augmenters and Reducers. Although 
A

SPECT statistical findings
L

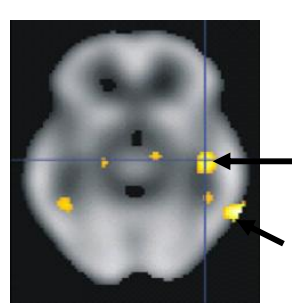

$\mathbf{R}$

A 22

BA 13

BA 37

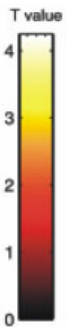

B

EEG

Background activity
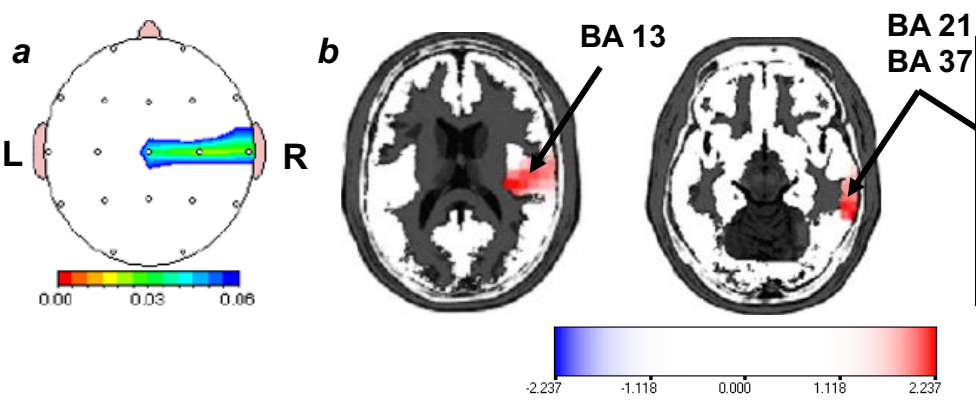

BA 21

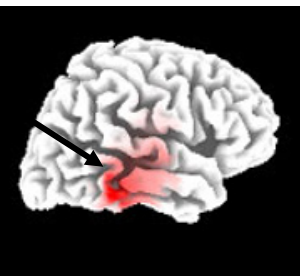

Figure 2. This figure presents statistical findings based on comparison between the groups: Augmenters and Reducers

A- SPECT statistical parametric maps showing areas of decreased $\mathrm{rCBF}$ in augmenters group at $\mathrm{z}>3.0$

B- Background EEG independent t- test findings showing increased theta absolute power in augmenters group. $a$ - Neuroguide $(\mathrm{p}<0.04) ; b$ - LORETA $(\mathrm{z}=2.3)$

BA 22- superior temporal gyrus; BA 21, BA 37- middle temporal gyrus;

BA 37 - inferior temporal gyrus BA 13- Insula

\section{SPECT and qEEG statistical findings}

Left

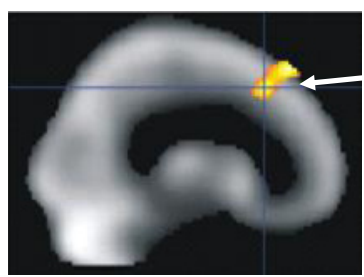

BA6

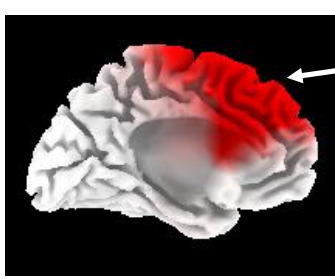

BA6 BA 8

BA9

BA32

BA24

BA33
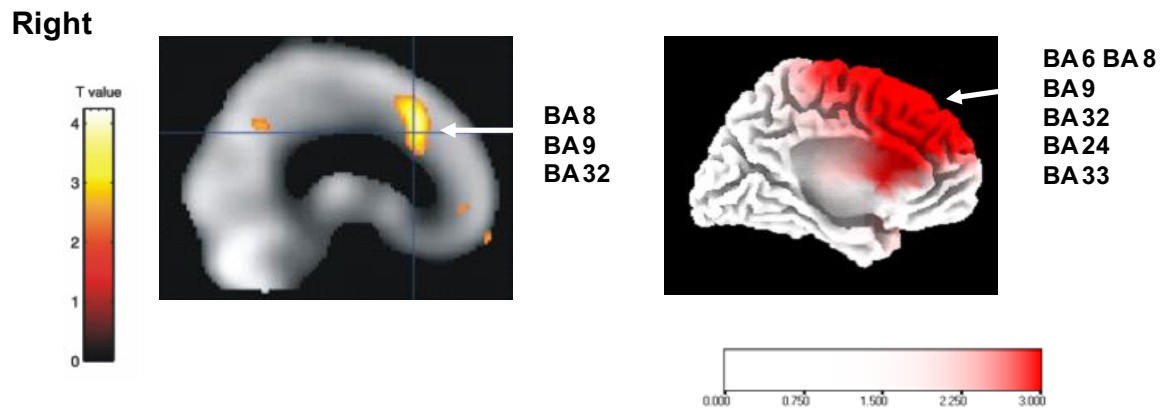

Figure 3. This figure presents SPECT statistical parametric maps showing areas of increased rCBF in Augmenters ( $\mathrm{z} \geq 3.0)$ and background EEG independent t- test findings showing increased theta absolute power in Augmenters $(\mathrm{z}=3.0)$. BA 6- superior frontal gyrus; BA 8, BA 9- medial frontal gyrus; BA 32, BA 24, BA 33- cingulate 
Table 2. The brain regions of statistically significant $(\mathrm{p}=0.02)$ findings in $\mathrm{rCBF}$ between Augmenters and Reducers

\begin{tabular}{|l|l|l|l|}
\hline Brain region & \multicolumn{1}{|l|}{$\begin{array}{l}\text { Talairach space } \\
\text { coordinates } \\
(\mathbf{x}, \mathbf{y}, \mathbf{z})\end{array}$} & $\begin{array}{l}\text { Cluster } \\
\text { volume (k) }\end{array}$ & z score \\
\hline \multicolumn{2}{|l|}{ Decreased rCBF in Augmenters in comparison with Reducers } \\
\hline $\begin{array}{l}\text { The right inferior temporal gyrus } \\
\text { (BA 37) }\end{array}$ & $64,-52,-10$ & 179 & 3.46 \\
\hline $\begin{array}{l}\text { The left middle temporal lobe } \\
\text { (white matter) }\end{array}$ & $-44,-46,0$ & 238 & 3.30 \\
\hline $\begin{array}{l}\text { The right middle temporal and } \\
\text { occipital gyrus (BA 39, BA 19) }\end{array}$ & $52,-76,10$ & 79 & 3.28 \\
\hline $\begin{array}{l}\text { The right superior temporal gyrus } \\
\text { (BA22) and insula (BA 13) }\end{array}$ & $44,-18,-8$ & 371 & 2.89 \\
\hline \multicolumn{1}{|l|}{ Increased rCBF in Augmenters in comparison with Reducers } \\
\hline $\begin{array}{l}\text { The left middle frontal gyrus, pre- } \\
\text { central gyrus (BA 6) }\end{array}$ & $-32,-8,48$ & 102 & 3.41 \\
\hline $\begin{array}{l}\text { The left middle frontal gyrus } \\
\text { (BA6) }\end{array}$ & $-16,38,56$ & 675 & 3.21 \\
\hline $\begin{array}{l}\text { The right superior frontal, medial } \\
\text { frontal gyrus, cingulate (BA 8, BA } \\
\text { 32) }\end{array}$ & $12,24,42$ & 165 & 3.14 \\
\hline $\begin{array}{l}\text { The right medial superior frontal } \\
\text { gyrus (BA11) }\end{array}$ & $6,62,-18$ & 511 & \\
\hline
\end{tabular}

the sample size was small, the significant differences found in baseline brain function between the PTSD subgroups are noteworthy and provide greater understanding of this disorder. We were able to differentiate patients with the same behavioral presentation into two biologically distinct groups. Further analysis indicated that the Augmenters endorsed more severe symptoms of PTSD. In addition, the augmenters' patterns of baseline brain abnormalities together with the pattern of the IDAEP response support the hypothesis that this PTSD subgroup would respond favorably to serotonin augmenting medications. Although, behaviorally similar, the PTSD subtype defined as Reducers endorses less severe PTSD symptoms and their response pattern to the IDAEP together with the baseline brain pathophysi- ology does not support the use of serotonergic medications in this PTSD subpopulation.

In our study, 29 (74\%) of 39 subjects had an augmenting response. These results are consistent with reports of augmenting response in a sample of 23 female Vietnam combat nurses (Metzger et al., 2002). An augmenting response was also reported by McPherson et al. (1997) who had shown that a group of 60 children of both genders diagnosed with PTSD showed greater amplitude of P2/N2 slopes than a non-PTSD group. Since the McPherson study included children and used different methodology (P2/N2 vs. N1/P2 slopes), we will not include this study in the following discussion. However, not all reports are in agreement with our findings. For example Paige, et al. (1990) showed that 9 (75\%) of 12 
Table 3. Statistical analysis (independent t-test) of behavioral measures between Augmenters and Reducers. Asterisk $(*)$ represents measures that reached statistical significance.

$C A P S$ - Clinician Administered PTSD Scale (CAPS- $B$ re-experiencing, $C A P S-C$ avoidance/numbing, and $C A P S-D$ increased arousal); $M-P T S D$ - Mississippi Scale for Combat related Post-Traumatic Stress Disorder;

BDI- Beck Depression Rating Scale; BDI- Question 16- question number 16 on Beck Depression Scale assessing sleep quality; STAIState-Trait Anxiety Inventory

\begin{tabular}{|c|c|c|c|c|}
\hline & & Mean & Standard Deviation & $P$ value \\
\hline CAPS-total * & $\begin{array}{l}\text { Augmenters } \\
\text { Reducers }\end{array}$ & $\begin{array}{l}79.4 \\
61.11\end{array}$ & $\begin{array}{l}14.230 \\
17.539\end{array}$ & 0.012 \\
\hline CAPS-B * & $\begin{array}{l}\text { Augmenters } \\
\text { Reducers }\end{array}$ & $\begin{array}{l}22.1 \\
14.89\end{array}$ & $\begin{array}{l}4.725 \\
6.809\end{array}$ & 0.008 \\
\hline CAPS- $C$ * & $\begin{array}{l}\text { Augmenters } \\
\text { Reducers }\end{array}$ & $\begin{array}{l}32.8 \\
26.22\end{array}$ & $\begin{array}{l}6.613 \\
8.983\end{array}$ & 0.043 \\
\hline CAPS-D * & $\begin{array}{l}\text { Augmenters } \\
\text { Reducers }\end{array}$ & $\begin{array}{l}24.5 \\
19.44\end{array}$ & $\begin{array}{l}5.911 \\
5.769\end{array}$ & 0.039 \\
\hline M-PTSD * & $\begin{array}{l}\text { Augmenters } \\
\text { Reducers }\end{array}$ & $\begin{array}{l}141.1 \\
126.56\end{array}$ & $\begin{array}{l}18.628 \\
11.555\end{array}$ & 0.028 \\
\hline BDI & $\begin{array}{l}\text { Augmenters } \\
\text { Reducers }\end{array}$ & $\begin{array}{l}30.2 \\
29.44\end{array}$ & $\begin{array}{l}14.062 \\
9.462\end{array}$ & 0.892 \\
\hline BDI- Question 16 * & $\begin{array}{l}\text { Augmenters } \\
\text { Reducers }\end{array}$ & $\begin{array}{l}1.30 \\
2.11\end{array}$ & $\begin{array}{l}0.300 \\
0.309\end{array}$ & 0.039 \\
\hline STAI-S & $\begin{array}{l}\text { Augmenters } \\
\text { Reducers }\end{array}$ & $\begin{array}{l}49.6 \\
52.11\end{array}$ & $\begin{array}{l}16.338 \\
7.801\end{array}$ & 0.671 \\
\hline STAI-T * & $\begin{array}{l}\text { Augmenters } \\
\text { Reducers }\end{array}$ & $\begin{array}{l}51.0 \\
62.0\end{array}$ & $\begin{array}{l}17.146 \\
6.764\end{array}$ & 0.043 \\
\hline
\end{tabular}

PTSD subjects were reducers and Lewine, et al. (2002) reported that $18(58 \%)$ out of 31 individuals diagnosed with PTSD had a reducing response pattern.

The literature on IDAEP in PTSD populations shows a high variability in response patterns. It appears that the IDAEP response pattern is influenced by gender, age, onset of PTSD relative to the time of the trauma, and the duration of symptoms. In addition, the number of studies and the total number of subjects involved in these studies are small which also contributes to reported discrepancies. Another reason for the discrepancies may be medication effects on the N1/P2 component of the auditory evoked potentials. The modulating effect of serotonergic medications on the IDAEP is described in the literature (Gallinat et al., 2000; Hegerl et al., 1998; Juckel et al., 2004; Tuchtenhagen, 2000). Of the four studies, including ours, investigating the adult PTSD IDAEP, only Paige et al. and Metzger et al. have studied medication-free subjects. Lewine et al. (2002) addressed the potential problem of medication effects by analyzing the IDAEP response differences between groups of subjects taking antidepressant, anxiolytic, and anticonvulsant medications. They reported no significant differences between the groups. The lack of statistical significance, however, could be due to the small number of subjects in each group: 17, 5, and 3 respectively.

Pharmacological interventions may modify IDAEP responses in individual patients. For this reason, we matched subjects in each group by medication types. It could be deduced that the degree of baseline neurotransmitter dysregulation among our PTSD subjects 
differed significantly, since only a portion of the tested subjects taking the same type of medications were able to demonstrate a reducing response pattern to the IDAEP. It is conceivable that the very strong augmenting responses were converted to more shallow responses and others may have been converted to reducers, as a result of the pharmacologic agents administration.

The current data support the hypothesis that differences in IDAEP processing may be reflected by resting brain states. The data analysis showed converging findings between the qEEG and SPECT that point to differences between the groups. Interestingly, increased theta band activity in Augmenters was found in brain areas with increased and decreased $\mathrm{rCBF}$ as compared to Reducers. In the Augmenters the converging findings were localized to the right temporal lobe and the insula, where increased theta activity and decreased $\mathrm{rCBF}$ were found as well as in the bilateral superior frontal gyri and the right anterior cingulate cortex, where increased theta activity was associated with increased rCBF.

The between-group differences in the right temporal theta activity suggest dysregulation of hippocampal function, since the hippocampus is involved in generation of theta activity (Vertes \& Kocsis, 1997). Interhemispheric differences in hippocampal structure and function in PTSD populations have been reported in the literature (Bremner, 2003; Bremner et al., 1997, 1999). Electrophysiological differences between the hemispheres have also been reported by Metzger et al. (2004) where the right parietal alpha asymmetry correlated with PTSD arousal symptoms. This finding again points to interhemispheric dysregulation in PTSD patients.

In addition to the hippopcampal system, theta activity is also generated in the anterior cingulate cortex (ACC). It has been postulated that theta rhythm may be generated in the ACC and hippocampus independently (Pizzagalli, Oakes, \& Davidson, 2003). The existence of two independent generators of theta activity explains the findings of increased theta activity in Augmenters in brain regions with increased (the superior frontal gyrus and anterior cingulate) and decreased (the right temporal lobe and insula) cerebral blood perfusion.

In a study with depressed subjects, the presence of higher theta activity in the rostral region of the anterior cingulate (BA 24 and BA 32) predicted better response to nortriptyline. Another study measuring treatment response based on baseline brain function showed that a PTSD population with increased baseline rCBF in the anterior cingulate showed favorable response to fluoxetine (Pizzagalli, 2001). These findings are in agreement with our results, since an augmenting response to the IDAEP is correlated with decreased central serotonergic activity. Therefore, it is expected that Augmenters but not Reducers would benefit from serotonin augmenting medications, notably SSRIs.
We also postulated that differences in baseline brain function would be manifested behaviorally. We obtained support for our hypothesis by findings of higher severity of PTSD core symptoms as measured by CAPS and M-PTSD in the Augmenters as compared with the Reducers. The higher PTSD symptom severity in subjects with an augmenting IDAEP response has also been reported by Metzger et al. (2002) and Lewine et al. (2002). Paige, et al. (1990), however, did not find a significant correlation between M-PTSD measures and the P2 slope.

The presence of a reducing pattern to the IDAEP may reflect a protective cortical state from sensory overload, as proposed in the literature (Paige et al., 1990; Pritchard, 1986; Schooler, Buchsbaum, \& Carpenter Jr, 1976). The presence of less intense PTSD symptoms in the reducers, as found in our study, would support this hypothesis.

The question is whether augmenters, who have more severe PTSD symptoms (based on CAPS total score, $\mathrm{p}=0.012$ ), have lost their protective capabilities over time or whether they ever had it developed. In the case of the patient population included in our study, the former may be true. These patients had long-standing PTSD histories and had been treated with multiple medications over the course of many years. This suggests that we might be dealing with a group of patients, whose brains have been depleted of their protective mechanisms, thus resulting in a treatment resistant PTSD population (resistant to the treatment administered according to the current treatment guidelines).

We also hypothesized that there might be different severity levels of co-morbidities between the two groups of PTSD patients. With regard to the total score of depression, neither group showed a statistically significant difference in symptom severity. After an item-by-item analysis of specific symptoms included in the BDI scale, we found that sleep disturbance was a differentiating variable between the two groups. Interestingly, the Reducers reported more sleep problems than the Augmenters. The worse sleep quality in Reducers was also positively correlated with the level of anxiety. Similar findings have been reported in patients with Generalized Anxiety Disorder. Higher anxiety levels in PTSD subjects with a reducing response to the IDAEP have been also reported by Paige, et al. (1990). He interpreted the reduction of the $\mathrm{P} 2$ slope as a protective inhibition in a response to anxiety. Our study supports the notion that PTSD comprises a heterogeneous group of patients. It provides evidence for the presence of two distinctly different PTSD patient populations, who, although similar in behavioral presentation, may require an individualized treatment approach. The limitations of this preliminary study, however, make the generalization of the reported findings to the entire PTSD population difficult. The main methodological limitation is 
the small sample size. Furthermore, since it was a naturalistic study, all subjects were treated with medications. In order to control for this variable, we matched medication types between the two groups. It is possible that we did not account for all potential medication effects, especially the variable length of exposure. It also leaves us with the unanswered question about the distribution of Augmenters and Reducers in unmedicated PTSD patient population.

\section{Acknowledgement}

Financial support came from the VA Research Service Merit Grant to Dr. Lukasz M. Konopka.

\section{REFERENCES}

APA Practice Guidelines (2004). http://www.psychiatryonline.com Arora, R. C., Fichtner, C. G., O'Connor, F., \& Crayton, J.W. (1993). Paroxetine binding in the blood platelets of posttraumatic stress disorder patients. Life Sciences, 53, 919-928.

Beck, A. T., Ward, C. H., Mendelson, M., Mock, J., \& Erbaugh, J. (1961). An inventory for measuring depression. Archives of General Psychiatry, 4, 561-571.

Blake, D. D., Weathers, F. W., Nagy, L. M., Kaloupek, D. G., Klauminzer, G., Charney, D. S., et al. (1990). A clinician rating scale for assessing current and lifetime PTSD: The CAPS-1. Behavior Therapist, 13, 187-188.

Bonne, O., Gilboa, A., Louzoun, Y., Brandes, D., Yona, I., Lester, H., et al. (2003). Resting regional cerebral perfusion in recent posttraumatic stress disorder. Biological Psychiatry, 54, 1077-1086.

Bremner, J. D. (2003). MRI and PET Study of Deficits in Hippocampal Structure and Function in Women With Childhood Sexual Abuse and Posttraumatic Stress Disorder. American Journal of Psychiatry, 160, 924-932.

Bremner, J. D., Narayan, M., Staib, L. H., Southwick, S. M., McGlashan, T., \& Charney, D. S. (1999). Neural correlates of memories of childhood sexual abuse in women with and without posttraumatic stress disorder. American Journal of Psychiatry, 156, 1787-1795.

Bremner, J. D., Randall, P., Vermetten, E., Staib, L., Bronen, R. A., Mazure, C., et al. (1997). Magnetic resonance imagingbased measurement of hippocampal volume in posttraumatic stress disorder related to childhood physical and sexual abuse-a preliminary report. Biological Psychiatry, 41, 23-32.

Buchsbaum, M. S. a. S. J. (1968). Stimulus intensity control and the cortical evoked response. Psychosomatic Medicine, $30,12-22$.

Foa, E. B., Davidson, J. \& Frances, A. (1999). The expert consensus guideline series: Treatment of posttraumatic stress disorder. Journal of Clinical Psychiatry, 60, 6-79.

Gallinat, J., Bottlender, R., Juckel, G., Munke-Puchner, A., Stotz, G., Kuss, H. J., et al. (2000). The loudness dependency of the auditory evoked N1/P2-component as a predictor of the acute SSRI response in depression. Psychopharmacology, 148, 404-411.

Harvey, B. H., Naciti, C., Brand, L., \& Stein, D. (2004). Serotonin and Stress: Protective or Malevolent Actions in the Biobehavioral Response to Repeated Trauma? Annals of the New York Academy of Sciences, 1032, 267.
Hegerl, U., Bottlender, R., Gallinat, J., Kuss, H. J., Ackenheil, M., \& Möller, H. J. (1998). The serotonin syndrome scale: first results on validity. European Archives of Psychiatry and Clinical Neuroscience, 248, 96-103.

Hegerl, U., Gallinat, J., \& Juckel, G. (2001). Event-related potentials Do they reflect central serotonergic neurotransmission and do they predict clinical response to serotonin agonists? Journal of Affective Disorders, 62, 93-100.

Juckel, G., Hegerl, U., Molnar, M., Csepe, V., \& Karmos, G. (1999). Auditory evoked potentials reflect serotonergic neuronal activity--a study in behaving cats administered drugs acting on 5-HT1A autoreceptors in the dorsal raphe nucleus. Neuropsychopharmacology, 21, 710-716.

Juckel, G., Mavrogiorgou, P., Bredemeier, S., Gallinat, J., Frodl, T., Schulz, C., et al. (2004). Loudness Dependence of Primary Auditory-Cortex-Evoked Activity as Predictor of Therapeutic Outcome to Prophylactic Lithium Treatment in Affective Disorders-A Retrospective Study. Pharmacopsychiatry, 37, 46-51.

Juckel, G., Molnár, M., Hegerl, U., Csépe, V., \& Karmos, G. (1997). Auditory-evoked potentials as indicator of brain serotonergic activity first evidence in behaving cats. Biological Psychiatry, 41, 1181-1195.

Keane, T. M. (1988). Mississippi Scale for Combat-Related Posttraumatic Stress Disorder: Three Studies in Reliabilty and Validity. Journal of Consulting and Clinical Psychology, 56, 85-90.

Lewine, J. D. (2002). Abnormal Stimulus-Response Intensity Functions in Posttraumatic Stress Disorder: An Electrophysiological Investigation. American Journal of Psychiatry, 159, 1689-1695.

Lucey, J. V., Costa, D. C., Adshead, G., Deahl, M., Busatto, G., Gacinovic, S., et al. (1997). Brain blood flow in anxiety disorders. OCD, panic disorder with agoraphobia, and posttraumatic stress disorder on 99mTcHMPAO single photon emission tomography (SPET). The British Journal of Psychiatry, 171, 346.

McPherson, W. B., Newton, J. E. O., Ackerman, P., Oglesby, D. M., \& Dykman, R. A. (1997). An event-related brain potential investigation of PTSD and PTSD symptoms in abused children. Integrative Psychological and Behavioral Science, 32, 31-42.

MEDx 3.4 .3 (1999). http://medx.sensor.com/

Metzger, L. J., Carson, M. A., Paulus, L. A., Lasko, N. B., Paige, S. R., Pitman, R. K., et al. (2002). Event-related potentials to auditory stimuli in female Vietnam nurse veterans with posttraumatic stress disorder. Psychophysiology, 39, 49-63.

Metzger, L.J., Paige, S.R., Carson, M.A., Lasko, N.B., Paulus, L.A., Pitman, R.K, \& Orr, S.P. (2004). PTSD arousal and depression symptoms associated with increased right-sided parietal EEG asymmetry. Jounal of Abnormal Psychology, 113, 324-9.

Mirzaei, S., Knoll, P., Keck, A., Preitler, B., Gutierrez, E., Umek, H., et al. (2001). Regional Cerebral Blood Flow in Patients Suffering from Post-Traumatic Stress Disorder. Neuropsychobiology, 43, 260-264.

Paige, S. R., Reid, G. M., Allen, M. G., \& Newton, J. E. (1990). Psychophysiological correlates of posttraumatic stress disorder in Vietnam veterans. Biol Psychiatry, 27(4), 419-430. Pascual-Marqui, R. D. (1994). Low resolution electromagnetic tomography. Brain Topography, 7, 180. 
Pizzagalli, D. (2001). Anterior Cingulate Activity as a Predictor of Degree of Treatment Response in Major Depression: Evidence From Brain Electrical Tomography Analysis. American Journal of Psychiatry, 158, 405-415.

Pizzagalli, D. A., Oakes, T. R., \& Davidson, R. J. (2003). Coupling of theta activity and glucose metabolism in the human rostral anterior cingulate cortex: An EEG/PET study of normal and depressed subjects. Psychophysiology, 40, 939949.

Pritchard, W. S. (1986). Cognitive event-related potential correlates of schizophrenia. Psychological bulletin., 100, 4366.

Sachinvala, N., Kling, A., Suffin, S., Lake, R., \& Cohen, M. (2000). Increased regional cerebral perfusion by $99 \mathrm{mTc}$ hexamethyl propylene amine oxime single photon emission computed tomography in post-traumatic stress disorder. Military Medicine, 165, 473-9.

Schooler, C., Buchsbaum, M. S., \& Carpenter Jr, W. T. (1976). Evoked response and kinesthetic measures of augmenting/reducing in schizophrenics: replications and extensions. Journal of Nervous and Mental Disease, 163, 221-232.

Southwick, S. M., Paige, S., Morgan 3rd, C. A., Bremner, J. D., Krystal, J. H., \& Charney, D. S. (1999). Neurotransmitter alterations in PTSD: catecholamines and serotonin. Seminars in Clinical Neuropsychiatry, 4, 242-248.

Spielberger, C. D., Gorsuch, R. L., \& Lushene, R. E. (1970). Manual for the State-Trait Anxiety Inventory. Palo Alto. Cal: Consulting Psychologists Press.

Talaraich, J., \& Tournoux, P. (1988). Co-Planar Stereotaxic Atlas of the Human Brain: New York: Thieme.

Tuchtenhagen, F. (2000). High Intensity Dependence of Auditory Evoked Dipole Source Activity Indicates Decreased Serotonergic Activity in Abstinent Ecstasy(MDMA) Users. Neuropsychopharmacology, 22, 608-617.

Vertes, R. P., \& Kocsis, B. (1997). Brainstem-diencephaloseptohippocampal systems controlling the theta rhythm of the hippocampus. Neuroscience, 81, 893-926. 